\title{
Clinical Evaluation after Introduction of Stem Cell Supernatant Biocell Shot 65 by Electroporation
}

\author{
Ryusuke Suzuki'1, Harue Suzuki², Maki Yokogawa3 ${ }^{3}$ Junichi Motohashi' \\ ${ }^{1}$ Division of Life Science and Engineering, School of Science and Engineering, Tokyo Denki University, Saitama, Japan \\ ${ }^{2}$ Suzuki Plastic Surgery Clinic, Kyoto, Japan \\ ${ }^{3}$ Yokogawa Dermatological Clinic, Kochi, Japan \\ ${ }^{4}$ Pure Code Inc., Osaka, Japan \\ Email: ryusuke.suzuki@mitsuichemicals.com
}

How to cite this paper: Suzuki, R., Suzuki, H., Yokogawa, M. and Motohashi, J. (2021) Clinical Evaluation after Introduction of Stem Cell Supernatant Biocell Shot 65 by Electroporation. Journal of Cosmetics, Dermatological Sciences and Applications, 11, 58-70.

https://doi.org/10.4236/jcdsa.2021.111006

Received: January 27, 2021

Accepted: March 27, 2021

Published: March 30, 2021

Copyright $\odot 2021$ by author(s) and Scientific Research Publishing Inc. This work is licensed under the Creative Commons Attribution International License (CC BY 4.0).

http://creativecommons.org/licenses/by/4.0/

\begin{abstract}
Objectives: The objective of this study is clinical evaluation after introduction of stem cell supernatant Biocell Shot 65 by electroporation. Background: There is a clinical report of psoriasis treatment by umbilical cord blood-derived stem cells, in which there was a beneficial result. These clinical results are the result of cord blood-derived cell growth factors. The umbilical cord blood-derived stem cell culture supernatant contains a high amount of TGF- $\beta$, PDGA, etc., and promotes wound healing. In addition, we suppose that the supernatant has the esthetic effect of improving skin condition, thus, we planned to make a clinical evaluation. However, it is difficult to set judgement criteria, and human judgement tends to be subjective. Therefore, in this study, as a new clinical evaluator, we used an AI skin diagnostic device with learning function. Methods: Stem cell culture supernatant Biocell Shot 65 was used as an introduction solution. After it was applied to the full faces of four patients, electroporation with BeBe pinocchio DM-5 SUPER DX Plus promoted infiltration deep in the keratinized layer. The patients were all healthy women in their forties. This procedure was performed every two weeks for three months. Wrinkles, fine lines around eyes, stains, pores, and $4 \mathrm{~V}$, which is considered as one diagnostic criteria, were evaluated at baseline and after each procedure with the skin diagnostic device HiMirror-Professional. In this study, macroscopic findings were not examined. Results: In all four patients, $4 \mathrm{~V}$ did not change remarkably. Patient 1 showed improvement after the procedure in wrinkles, fine lines around eyes, stains, and pores. Patient 2 revealed improvement only in fine lines around eyes, but did not show improvement in other items. Patient 3 improved in wrinkles, stains, and pores
\end{abstract}


only after the second procedure, but did not show improvement in the other procedures. Patient 4 improved in all items except $4 \mathrm{~V}$ after the fourth procedure. In addition, when the mean values of all the patients were plotted and compared before and after each procedure, there was a trend toward improvements after the fifth procedure. Conclusion: In addition to application of supernatant culture to the skin, introduction of the solution to the skin by electroporation might improve stains, wrinkles, and pores. Our evaluation was performed with a facial diagnostic device three months after the start of the procedure.

\section{Keywords}

Clinical Evaluation, Stem Cell, Skin Treatment

\section{Introduction}

There is a clinical report about umbilical cord blood-derived stem cell treatment for a patient with psoriatic arthritis, the result of which was beneficial [1]. Margaret Coutts et al. conclude that stem cells in umbilical cord blood support tissue repair and suppress over-inflammation. There is another report about tissue repair support and over-inflammation suppression by umbilical cord blood-derived stem cells [2]. It is a well-known fact that umbilical cord blood-derived stem cells have some effects in the body. Cells in the body produce many cell growth factors for cell growth, cell migration, and immunosuppression, etc. Various treatments by cell growth factors are applied in clinical and esthetic practice based on this principle [3]. Transdermal application of one of the growth factors or direct introduction to the wound site stimulates the body's healing abilities, and is beneficial even in persistent injury such as intractable skin ulcer. Many cell growth factor agents, however, use a single growth factor, and they do not sufficiently affect the wound site due to protease in the wound site or absorption by gauze. Therefore, several applications to the wound site are required per day. Kuroyanagi et al. used a wound dressing made of hyaluronic acid and collagen with EGF in order to prevent inactivation of growth factors by degrading enzymes. This dressing decreased the number of applications and the financial burden of the patients, and improved wound healing [4]. Based on these reports, we supposed that cell growth factors in umbilical cord blood-derived stem cells would support tissue repair and suppress over-inflammation. In order to use these growth factor agents on a daily basis, they should be used as a cosmetic. Patients can take care of their skin daily at home, and non-severe skin damage such as photoaging can be handled effectively by cosmetics. Mesenchymal stem cells are pluripotent cells, which originate in various tissues such as bone marrow, fat tissue, and umbilical cord blood. We used umbilical cord blood-derived mesenchymal stem cells because they have more biological advantages than other $\mathrm{Me}$ - 
senchymal stem cells [5] [6] [7]. Mesenchymal stem cells are essential for regeneration of the skin, so they are the most important cells for the skin [8]. Recent studies suggested that Mesenchymal stem cells stimulate human dermal fibroblasts through a paracrine mechanism, and promote the wound healing in the skin [9] [10]. Mesenchymal stem cells secrete many cytokines and growth factors such as epidermal growth factor (EGF), fibroblast growth factor (bFGF), and transforming growth factor $\beta$ (TGF- $\beta$ ). These are important for cell growth and skin maintenance [11] [12]. In our study, we used umbilical cord blood-derived stem cell culture supernatant, which is rich in TGF- $\beta$. TGF- $\beta$ is involved in the wound healing process such as inflammation, angiogenesis, fibroblast growth, collagen synthesis and deposition, and extracellular matrix remodeling [13] [14]. Umbilical cord blood-derived stem cell culture supernatant also contains Platelet-Derived Growth Factor (PDGF) as a main ingredient, which activates many metabolic processes such as synthesis of protein and collagen, collagenase activity, migration of fibroblasts and smooth muscle cells [15]. Since these growth factors are expected to improve skin such as age spots, wrinkles, and pores, we examined the effect of cosmetic Biocellshot 65. In addition to application of culture supernatant to the skin, in this study, introduction to the skin by electroporation was performed. The results were evaluated with the facial diagnostic device during three months after the start of the procedure.

\section{Materials and Methods}

\subsection{Introduction Solution (Biocell Shot 65)}

Biocell Shot 65 (Pure Code Inc.) contains more than 300 growth factors. In contrast, the usual agents contain only a single growth factor (see Figure 1). EGF is, for example, widely used in China as a burn medication or decubitus treatment. Biocell Shot 65 also contains EGF. A single application of EGF to the skin promotes the growth of epidermal cells and fibroblasts, and production of Vascular Endothelial Growth Factor (VEGF) and Hepatocyte Growth Factor (HGF) [4]. Biocell Shot 65 is expected to affect the skin in various ways because it contains many growth factors which have beneficial effects on the skin even with a single factor. In particular, TGF- $\beta 1$ and PDGF-AA are contained in units of $\mathrm{ng} / \mathrm{mL}$, so they are expected to have a remarkable effect. TGF- $\beta 1$ promotes production and deposition of extracellular matrix, and has an anti-inflammatory effect. Furthermore, it is supposed to have an anti-cancer effect, and was found first in the 33 members of the TGF family. TGF mainly acts as a modulator, and in this solution containing many growth factors, it is essential as a modulator. PDGF-AA activates hair papilla, and will work as a hair growth agent. PDGF also acts as a modulator, and is expected to suppress cell overgrowth and tumor development, with beneficial effects to modulate other growth factors. Therefore, we introduced this solution to the patients, $1 \mathrm{~mL}$ per application. In addition, in order to carry out the introduction more smoothly, SEREM MORE POTELE was used in combination as a solvent for Biocell Shot 65 at the same time. 


\subsection{Introduction Device (BeBe Pinocchio DM-5 SUPER DX Plus)}

BeBe pinocchio DM-5 SUPER DX Plus (Pure Code Inc.) is an electroporation device used on the skin (Figure 2). As a principle of electroporation, a sigmoidal pulse of less than $60 \mathrm{~V}$ leads to electrical breakdown in the keratinized layer of the skin. Immediately after the treatment, pores are observed $0.2 \mathrm{~mm}$ in diameter, and $0.03 \mathrm{~mm}$ in diameter after 5 minutes. After 10 minutes, pores close back to the original state. A high voltage of $25,000 \mathrm{~V}$ per $\mathrm{cm}^{2}$ causes electrical breakdown and these phenomena. Also, pores in cell membranes open, so various substances can infiltrate into the skin cells [16]. Harue Suzuki et al. proved that hyaluronic acid of molecular weight 1,000,000 penetrates the skin with this device, therefore, the ingredients of stem cell supernatant can be introduced into skin cells with the device [17].

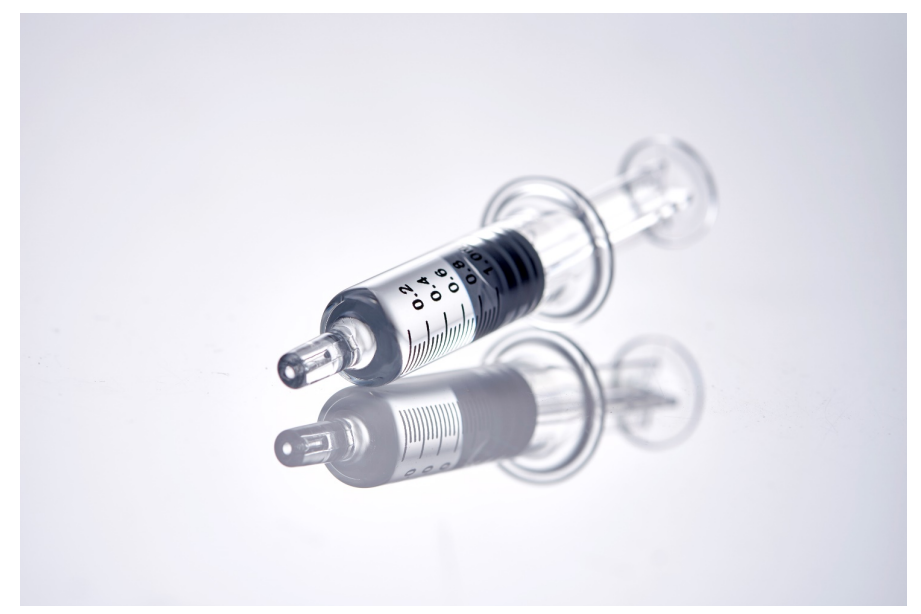

Figure 1. Biocell Shot 65. The concentration of umblilical cord blood-derived stem cell culture supernatant is $65 \%$ in this solution. $1 \mathrm{~mL}$ is applied to the full face.

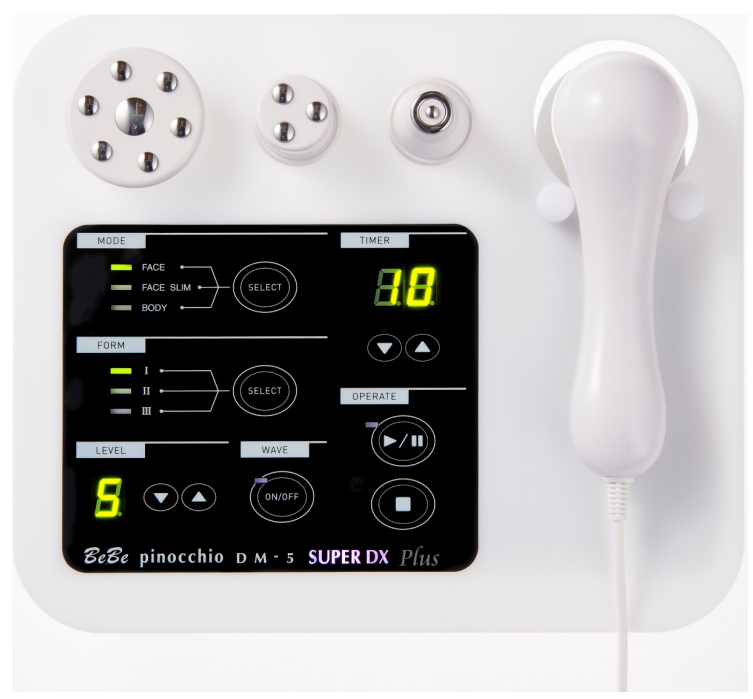

Figure 2. BeBe pinocchio DM-5 SUPER DX Plus. The device has a Cytoplasm Pass System, which introduces macromolecules and unionized neutral molecules into the dermal skin without a needle. The three types of sockets are used for body, face, and spot. 


\subsection{Face Diagnostic Device (HiMirror-Professional)}

HiMirror-Professional (B-by-C Inc.) is a measurement device which quantifies facial proportion with AI (Figure 3 ). It can measure and quantify stains, wrinkles, pores, etc. In this study, the actual measurement was compared with the mean ideal values by age which were calculated from the skin data of 20,000 people. The difference between the ideal value and the actual value was plotted, and a small difference represents the ideal condition. In this study, stains, wrinkles, fine lines, pores, and $4 \mathrm{~V}$ were evaluated. $4 \mathrm{~V}$ is a specific facial area, measured from a front photo of the face. By measuring this area, the facial proportion is quantified. It is expressed by a zone V1 where the center is the cheek, and a zone V2 where the center is the sides of the mouth. Their ratio determines the facial proportion. The cosmetic results were conventionally evaluated by impression, but $4 \mathrm{~V}$ is one of the markers which quantifies the cosmetic result.

\subsection{Clinical Trial}

The age of patients, number and content of the procedures are listed in Table 1. The patients spent their daily life in the usual way without diet or sleep restrictions. Patients were extracted from the patients visiting the clinic. Since the biocell

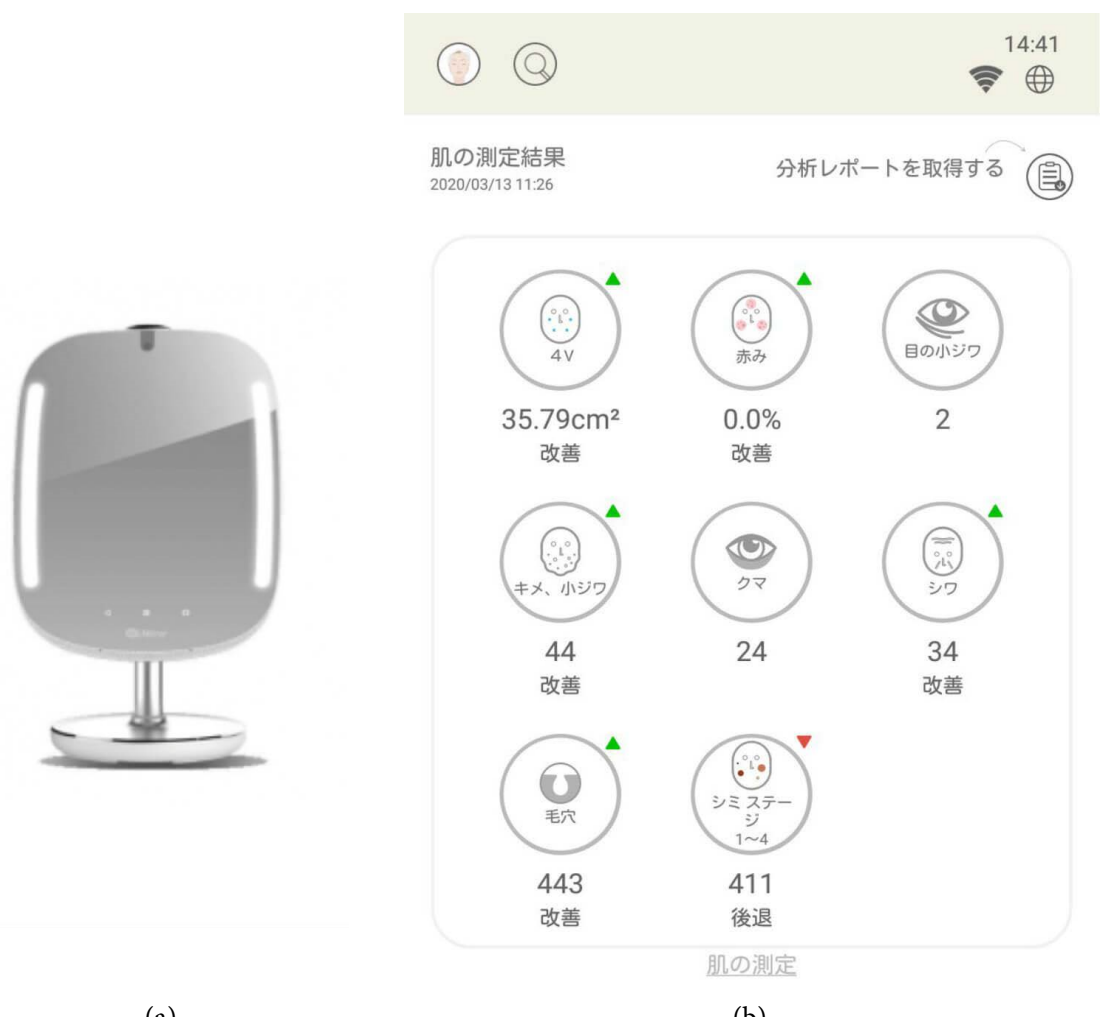

(b)

Figure 3. HiMirror-Professional. (a) HiMirror-Professional. HiMirror Professional uses the photos of patients, analyzes the skin, and saves the data. It can acquire daily skin data, and care for especially necessary sites; (b) Diagnosis. Seven items are measured; stains, pores, wrinkles, fine lines, roughness, redness, and dark circles. 
shot 65 used is a cosmetic product, this experiment was performed according to the guidelines established by Pure Code Co., Ltd. The patients were selected from those who agreed to this experiment among the patients who visited the clinic and those who were healthy and had no serious illness. If abnormal reactions such as skin redness were observed during the trial, it was planned to discontinue it.

\subsection{Statistical Analysis}

Significant differences were obtained using the $t$ test for equi-dispersive data, and unequal variance using the Welch $t$ test. A $p$ value less than 0.05 was considered statistically significant.

\section{Results}

The values for each patient were plotted (Figure 4). The facial photos of Patient 1 and 2 are shown with their permission (Figure 5). Wrinkles, fine lines around eyes, stains, and pores in Patient 1 improved after the procedure. $4 \mathrm{~V}$ of Patient 1 did not change. Patient 2 said that she felt the stimulation near the eyelids during the third and fifth procedure because the voltage of the device was high. However, abnormal reactions such as skin redness were not observed, so it did not affect the subsequent procedures. Fine lines around eyes improved in Patient 2 , but there was no change in other items. In Patient 3, wrinkles, stains, and pores improved after the second procedure, but did not change afterwards compared with the previous skin. In Patient 4 , all items except $4 \mathrm{~V}$ improved after the fourth procedure. The mean values of all patients were plotted before and after the procedures, and there was a trend toward improvement after the procedure. However, there was no significant difference $(\mathrm{p}>0.05)$ (Figure 6).

Table 1. Patient background and the procedure.

\begin{tabular}{|c|c|c|c|c|c|c|c|}
\hline & Gender & Age & $\begin{array}{l}\text { Device for introducing } \\
\text { solution }\end{array}$ & Solution & $\begin{array}{l}\text { Measurement } \\
\text { device }\end{array}$ & $\begin{array}{l}\text { Duration of } \\
\text { procedures }\end{array}$ & $\begin{array}{l}\text { Number of } \\
\text { procedures }\end{array}$ \\
\hline Patient 1 & female & 45 y.o. & $\begin{array}{l}\text { BeBe pinocchio DM-5 } \\
\text { SUPER DX Plus }\end{array}$ & $\begin{array}{c}M+\text { Biocell } \\
\text { Shot } 65\end{array}$ & HiMirror-Professional & $\begin{array}{c}\text { March 24-June 13, } \\
2020\end{array}$ & 5 \\
\hline Patient 2 & female & 49 y.o. & $\begin{array}{l}\text { BeBe pinocchio DM-5 } \\
\text { SUPER DX Plus }\end{array}$ & $\begin{array}{l}M+\text { Biocell } \\
\quad \text { Shot } 65\end{array}$ & HiMirror-Professional & $\begin{array}{l}\text { April 4-August 1, } \\
2020\end{array}$ & 4 \\
\hline Patient 3 & female & 46 y.o. & $\begin{array}{l}\text { BeBe pinocchio DM-5 } \\
\text { SUPER DX Plus }\end{array}$ & $\begin{array}{l}M+\text { Biocell } \\
\text { Shot } 65\end{array}$ & HiMirror-Professional & $\begin{array}{l}\text { June 2-August 5, } \\
\qquad 2020\end{array}$ & 5 \\
\hline Patient 4 & female & 48 y.o. & $\begin{array}{l}\text { BeBe pinocchio DM-5 } \\
\text { SUPER DX Plus }\end{array}$ & $\begin{array}{l}M+\text { Biocell } \\
\text { Shot } 65\end{array}$ & HiMirror-Professional & $\begin{array}{l}\text { May 16-July 11, } \\
2020\end{array}$ & 5 \\
\hline
\end{tabular}




\section{Patient 1}

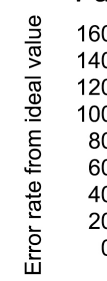
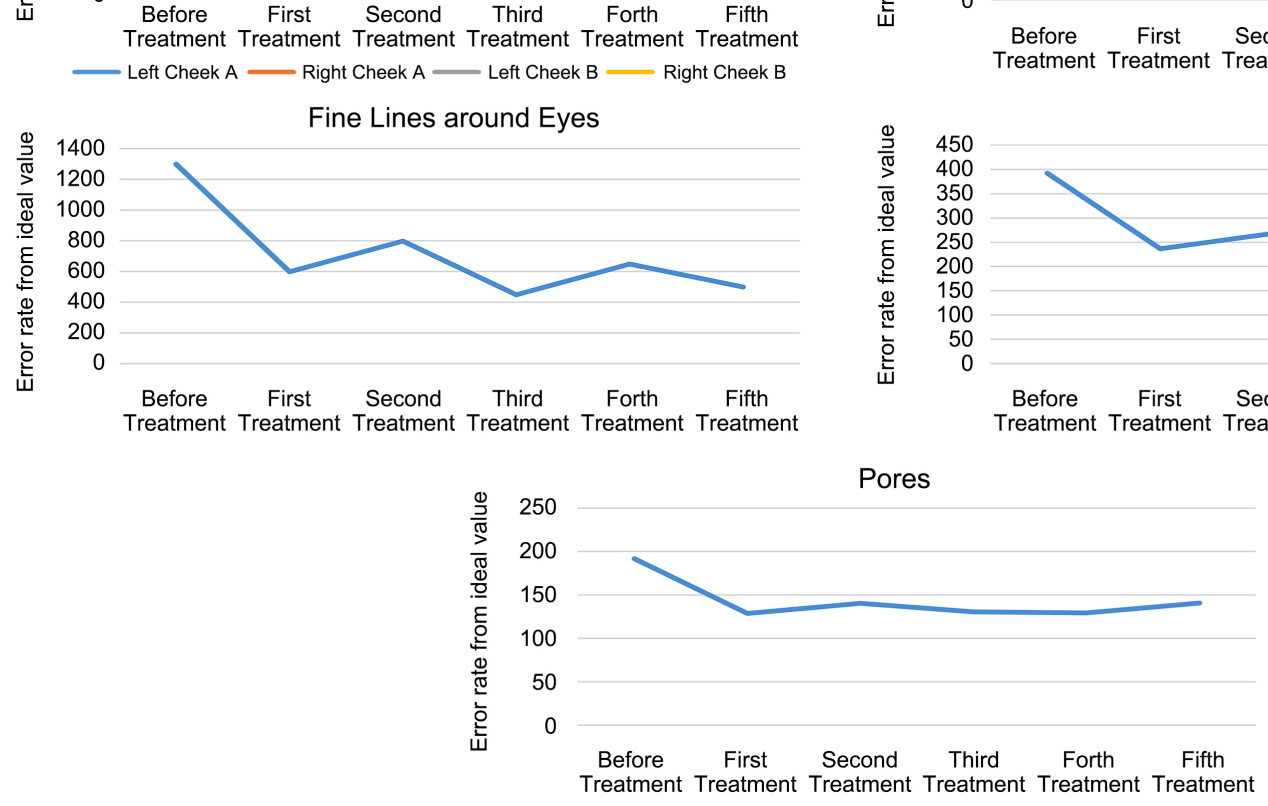

(a)
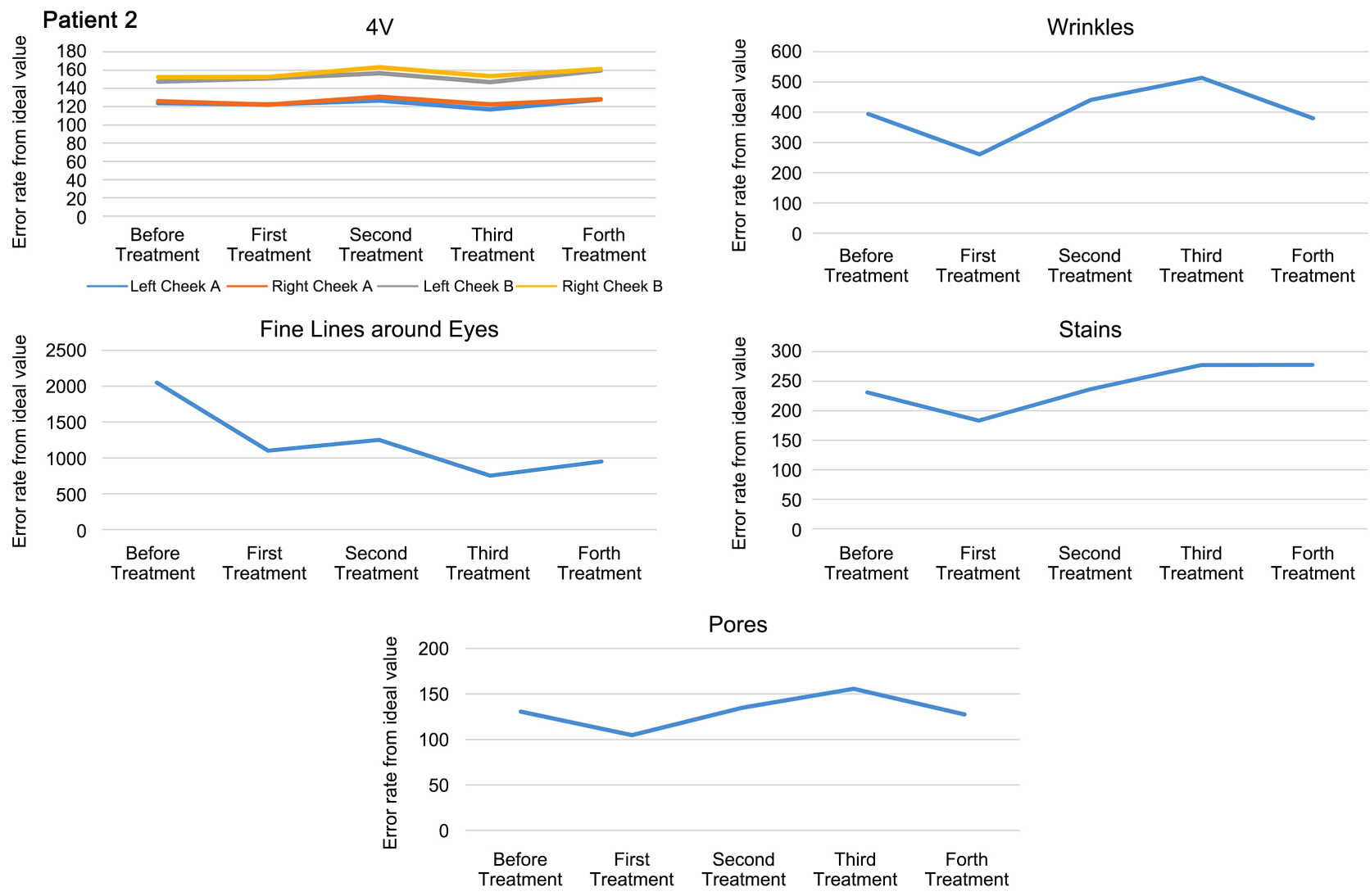

(b) 


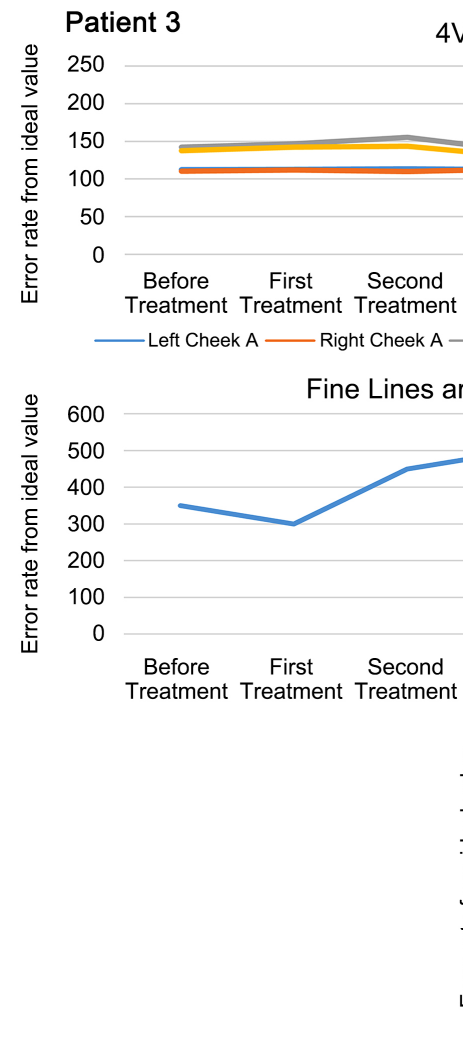

4V

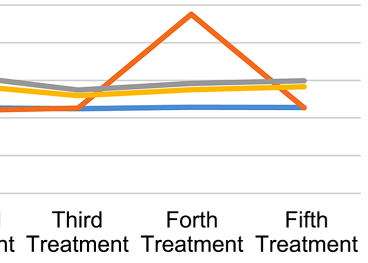

Left Cheek B — Right Cheek B
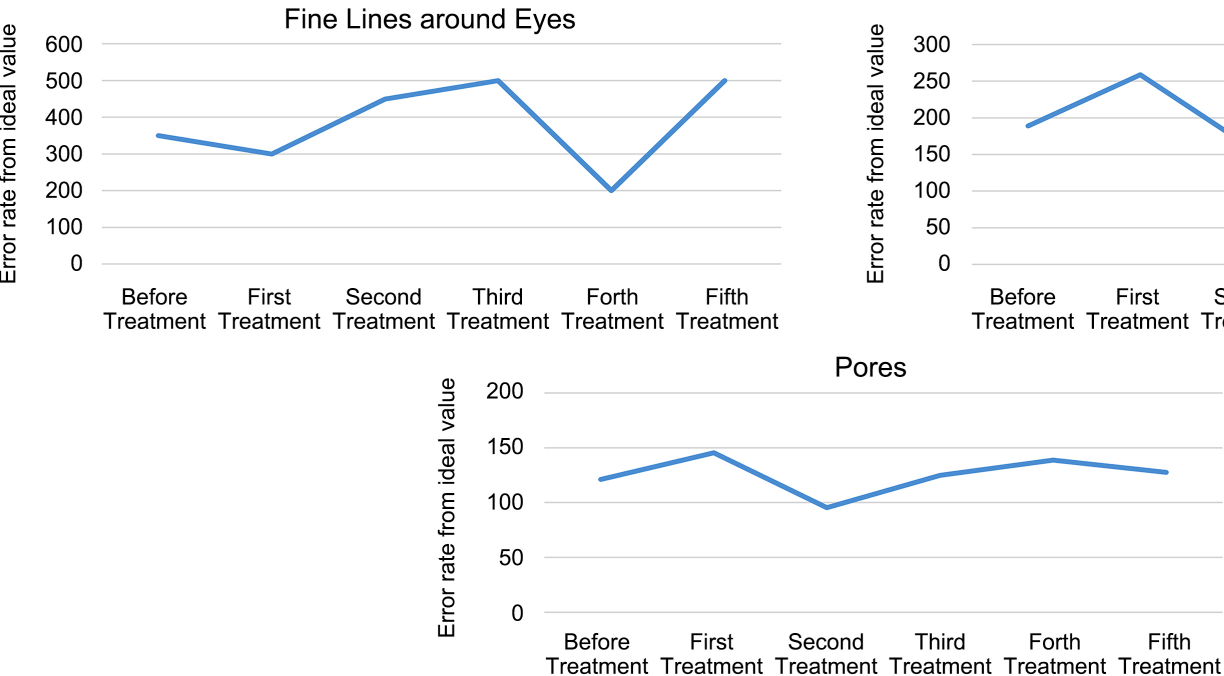

(c)
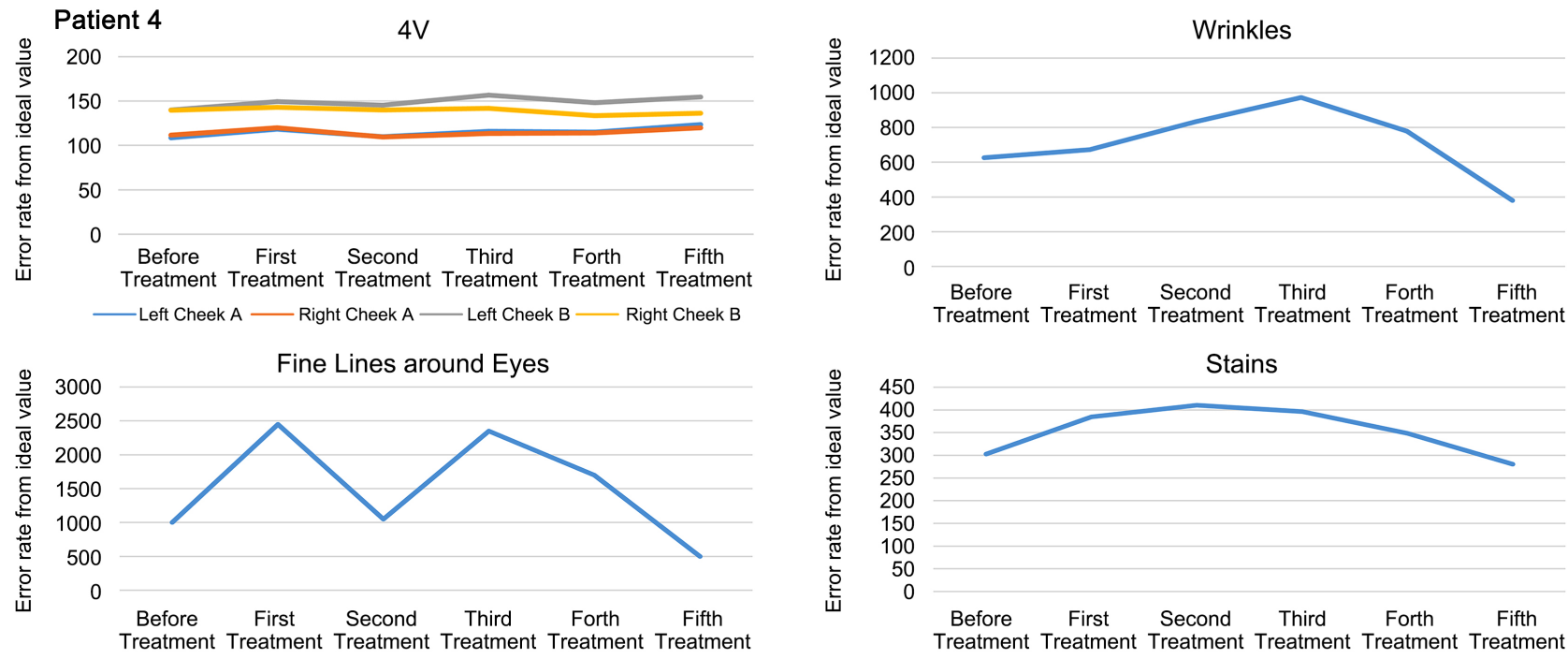

Pores

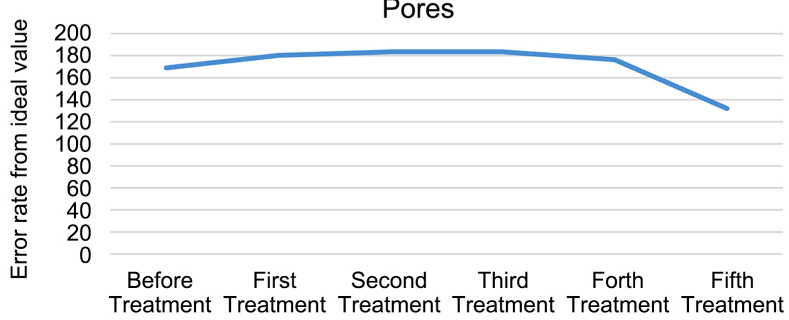

(d)

Figure 4. Results. 4V, wrinkles, fine lines, stains, and pores were measured. (a) Patient 1; (b) Patient 2; (c) Patient 3; (d) Patient 4. 


\section{Before treatment}

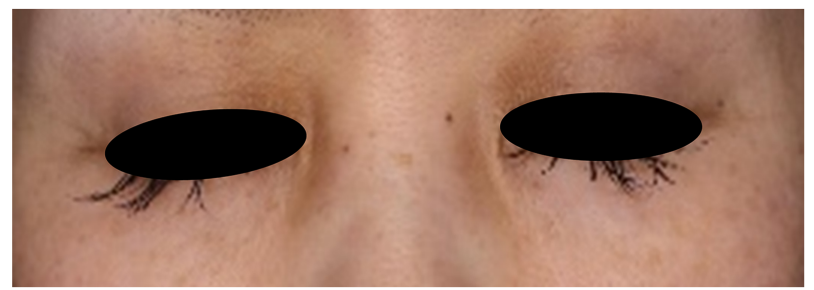

1st treatment

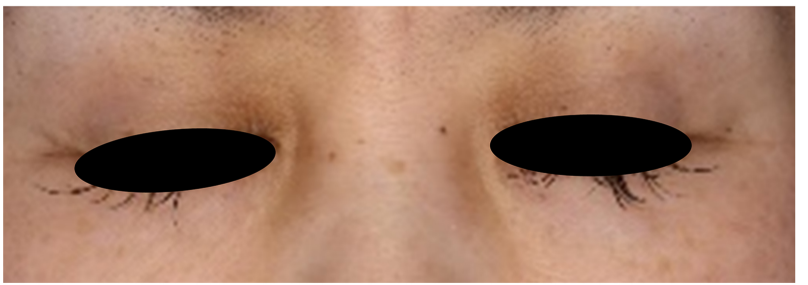

\section{2nd treatment}

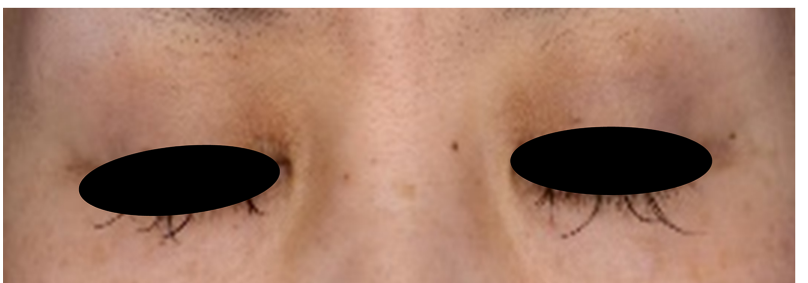

\section{Before treatment}

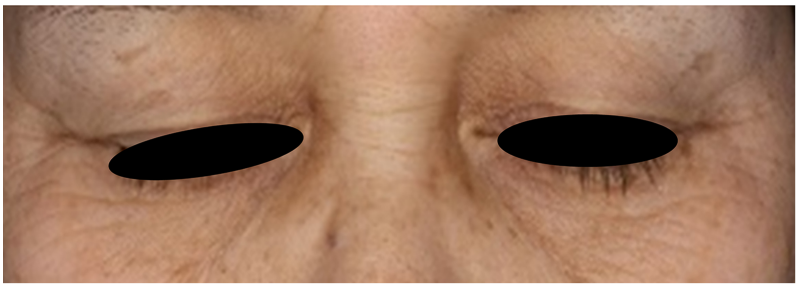

\section{1st treatment}

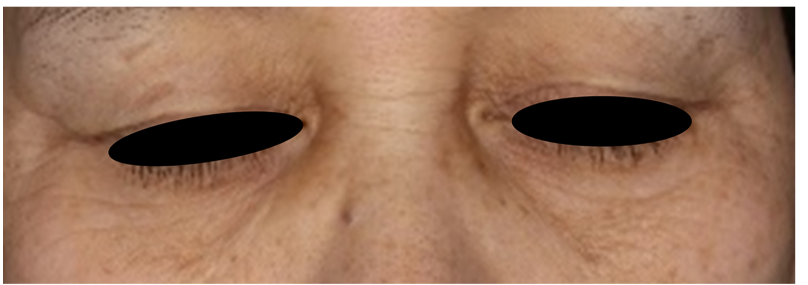

\section{2nd treatment}

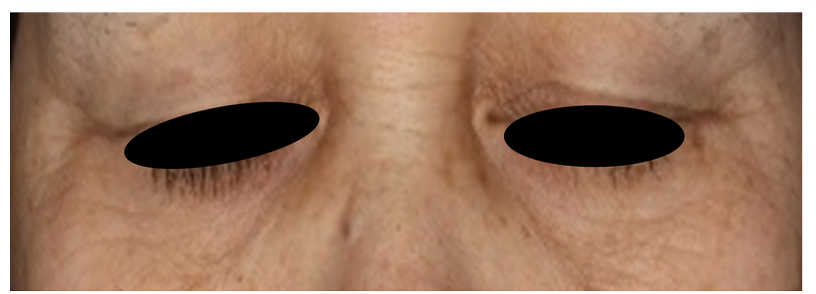

(b) 3rd treatment

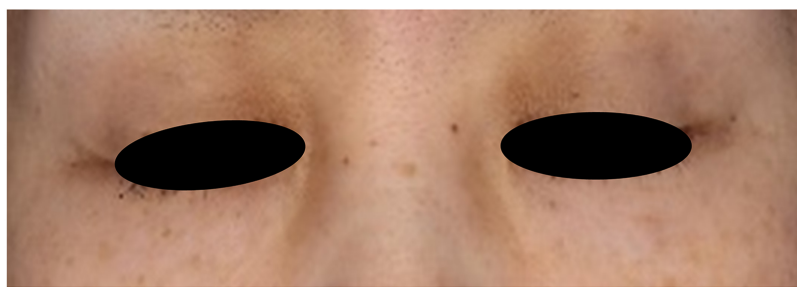

4th treatment

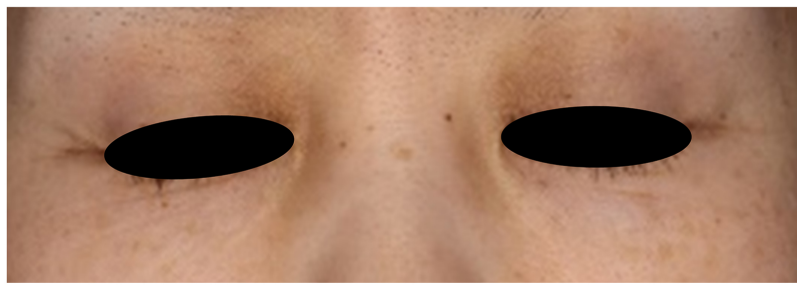

5th treatment

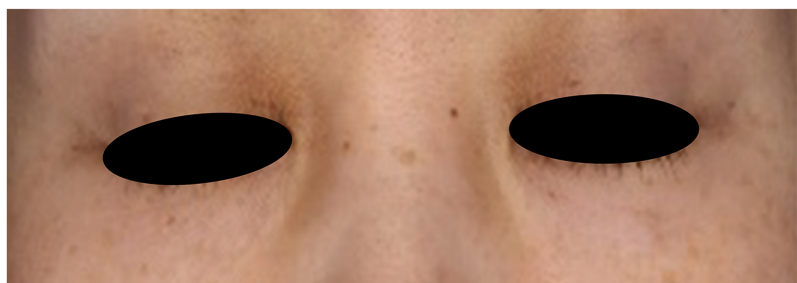

(a)

\section{3rd treatment}

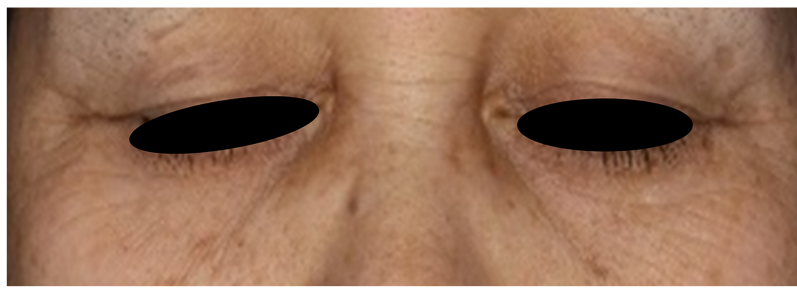

4th treatment

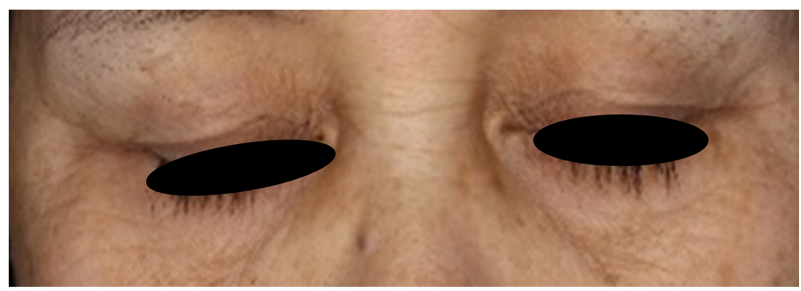

Figure 5. Photo evaluation. (a) Patient 1; (b) Patient 2. 


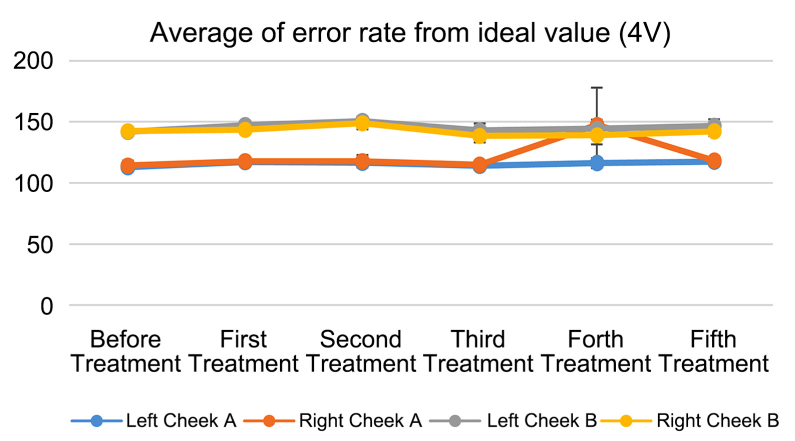

(a)

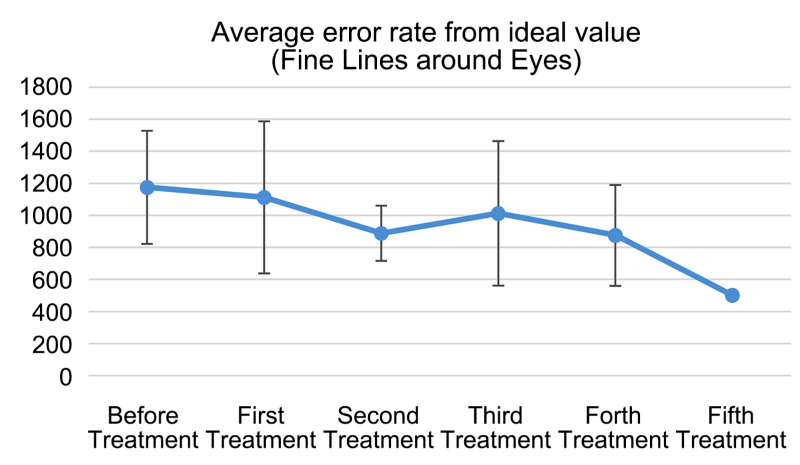

(c)

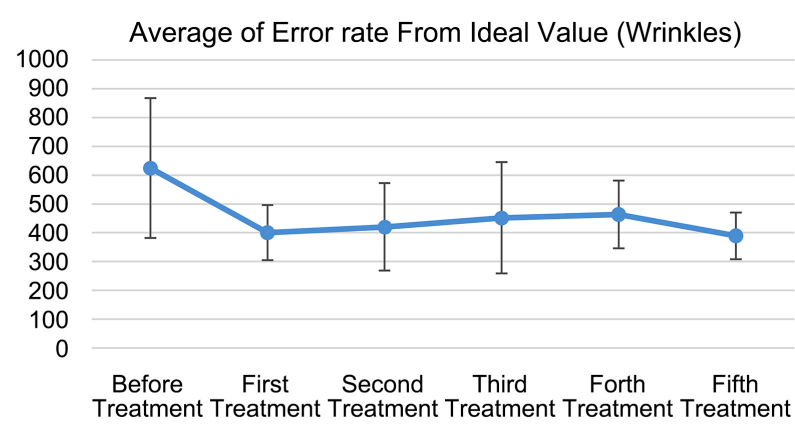

(b)

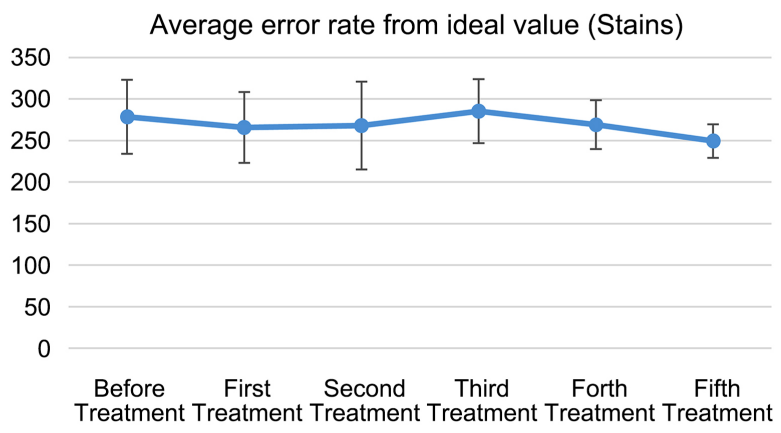

(d)

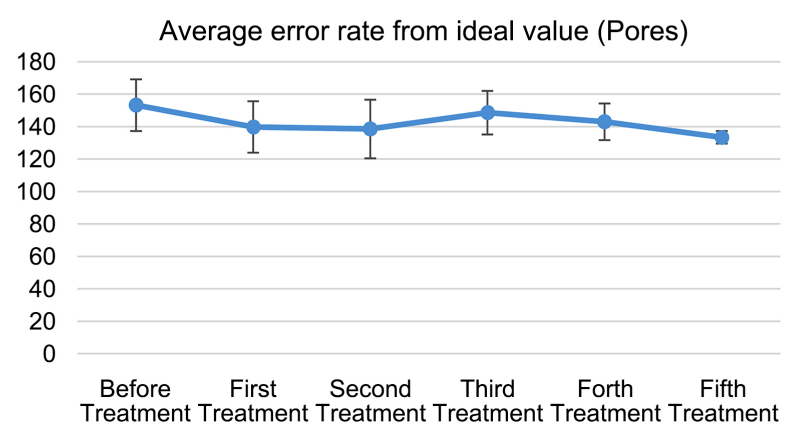

(e)

Figure 6. Efficacy of the procedure. Difference between the mean value and the ideal value in 4 patients at baseline and after each procedure. (a) 4V; (b) wrinkles; (c) fine lines; (d) stains; (e) pores.

\section{Discussion}

The stem cell culture supernatant which we used in this study contains a high concentration of TGF- $\beta$ and PDGF-AA. These growth factors are expected to be introduced into the inner skin by electroporation. The molecular weight of the growth factors are $25 \mathrm{kDa}$ and $28.9 \mathrm{kDa}$ respectively, and the usual pore size of the skin is $50 \mathrm{~nm}$, so they can be introduced by the device in this study. They are expected to act on epidermal cells and cause cascade reactions. This means that epidermal cells receive various growth factors, then, the epidermal cells produce other growth factors, activate fibroblasts and endothelial cells in the dermal layer, and promote wound healing. We performed not only simple application to the skin but also electroporation, because we needed to confirm that cascade 
reactions plus direct introduction of active ingredients to the dermal layer can promote collagen production. In this study, improvement of wrinkles in one month was confirmed macroscopically, and this was not done just by simple application. In the future, the introduction device and solution will be applied to a hair growth procedure. This is because the pore size of hair papilla is $300 \mathrm{~nm}$, larger than the pore size of the skin, so the active ingredients can be introduced to the target site more easily. Change of the active ingredients and introduction site might lead to more effective results. In particular, for stains, introduction of anti-oxidants such as vitamin $\mathrm{C}$ derivatives and resveratrol, and whitening ingredients such as ascorbic acid glucoside and tranexamic acid, can cure stains more effectively. For safety, this study was performed in two once a week procedures, then every two weeks, but three or four once a week procedures, then every 10 days or every two weeks, might be better, according to the therapist. After confirming that there were no allergic reactions, more frequent use might lead to more effective results. In this study, macroscopic findings were not included in order to eliminate subjective judgement, and the items were evaluated by the skin diagnostic device with AI. This diagnostic device contains mean values and ideal values based on the skin data of approximately 20,000 people. It also has AI, and when it acquires new data, the values are renewed and the data quality improves. As data quality improves, more precise data will be expected.

\section{Conclusion}

In addition to application of culture supernatant to the skin, introduction to the skin by electroporation might improve stains, wrinkles, and pores. Our evaluation was performed with a facial diagnostic device three months after the start of the procedure.

\section{Conflicts of Interest}

The authors declare no conflicts of interest regarding the publication of this paper.

\section{References}

[1] Coutts, M., Soriano, R., Naidoo, R. and Torfi, H. (2017) Umbilical Cord Blood Stem Cell Treatment for a Patient with Psoriatic Arthritis. World Journal of Stem Cells, 9 235-240. https://doi.org/10.4252/wjsc.v9.i12.235

[2] Rizk, M., Aziz, J., Shorr, R. and Allan, D.S. (2017) Cell-Based Therapy Using Umbilical Cord Blood for Novel Indications in Regenerative Therapy and Immune Modulation: An Updated Systematic Scoping Review of the Literature. Biology of Blood and Marrow Transplantation, 23, 1607-1613. https://doi.org/10.1016/j.bbmt.2017.05.032

[3] Yu, A., Takeda, A., Kumazawa, K., Miyoshi, H., Kuroyanagi, M., Yoshitake, T., Uchinuma, E., Suzuki, R. and Kuroyanagi, Y. (2015) Preliminary Clinical Study Using a Novel Wound Dressing Composed of Hyaluronic Acid and Collagen Containing EGF. Open Journal of Regenerative Medicine, 4, 6-13.

[4] Jin, H.J., Bae, Y.K., Kim, M., Kwon, S.J., Jeon, H.B., Choi, S.J., Kim, S.W., Yang, 
Y.S., Oh, W. and Chang, J.W. (2013) Comparative Analysis of Human Mesenchymal Stem Cells from Bone Marrow, Adipose Tissue, and Umbilical Cord Blood as Sources of Cell Therapy. International Journal of Molecular Sciences, 14, 17986-18001. https://doi.org/10.3390/ijms140917986

[5] Kern, S., Eichler, H., Stoeve, J., Kluter, H. and Bieback, K. (2006) Comparative Analysis of Mesenchymal Stem Cells from Bone Marrow, Umbilical Cord Blood, or Adipose Tissue. Stem Cells, 24, 1294-1301. https://doi.org/10.1634/stemcells.2005-0342

[6] Ding, D.C., Chang, Y.H., Shyu, W.C. and Lin, S.Z. (2015) Human Umbilical Cord Mesenchymal Stem Cells: A New Era for Stem Cell Therapy. Cell Transplant, 24, 339-347. https://doi.org/10.3727/096368915X686841

[7] Rivers, J.K. (2014) Stem Cells for Skin Rejuvenation: Are We There Yet? Journal of Cutaneous Medicine and Surgery, 18, 75-76.

https://doi.org/10.2310/7750.2014.EDIT18.2

[8] Kim, W.S., Park, B.S., Sung, J.H., Yang, J.M., Park, S.B., Kwak, S.J. and Park, J.S. (2007) Wound Healing Effect of Adipose-Derived Stem Cells: A Critical Role of Secretory Factors on Human Dermal Fibroblasts. Journal of Dermatological Science, 48, 15-24. https://doi.org/10.1016/j.jdermsci.2007.05.018

[9] Sasaki, M., Abe, R., Fujita, Y., Ando, S., Inokuma, D. and Shimizu, H. (2008) Mesenchymal Stem Cells Are Recruited into Wounded Skin and Contribute to Wound Repair by Trans Differentiation into Multiple Skin Cell Type. The Journal of Immunology, 180, 2581-2587. https://doi.org/10.4049/jimmunol.180.4.2581

[10] Kim, W.S., Park, B.S., Park, S.H., Kim, H.K. and Sung, J.H. (2009) Antiwrinkle Effect of Adipose-Derived Stem Cell: Activation of Dermal Fibroblast by Secretory Factors. Journal of Dermatological Science, 53, 96-102.

https://doi.org/10.1016/j.jdermsci.2008.08.007

[11] Yoon, B.S., Moon, J.H., Jun, E.K., Kim, J., Maeng, I., Kim, J.S., Lee, J.H., Baik, C.S., Kim, A., Cho, K.S., Lee, J.H., Lee, H.H., Whang, K.Y. and You, S. (2010) Secretory Profiles and Wound Healing Effects of Human Amniotic Fluid-Derived Mesenchymal Stem Cells. Stem Cells and Development, 19, 887-902. https://doi.org/10.1089/scd.2009.0138

[12] Roberts, A.B., Sporn, M.B., Assoian, R.K., Smith, J.M., Roche, N.S., Wakefield, L.M., Heine, U.I., Liotta, L.A., Falanga, V., Kehrl, J.H. and Fauci, A.S. (1986) Transforming Growth Factor- $\beta$ : Rapid Induction of Fibrosis and Angiogenesis in Vivo and Stimulation of Collagen Formation in Vitro. Proceedings of the National Academy of Sciences of the United States of America, 83, 4167-4171.

https://doi.org/10.1073/pnas.83.12.4167

[13] Nall, A.V., Brownlee, R., Colvin, C.P., Schultz, G., Fein, D., Cassisi, N.J., Nguyen, T. and Kalra, A. (1996) Transforming Growth Factor Beta 1 Improves Wound Healing and Random Flap Survival in Normal and Irradiated Rats. Archives of Otolaryngology, 122, 171-177. https://doi.org/10.1001/archotol.1996.01890140057011

[14] Nixont, C., et al. (1987) Role of Platelet-Derived Growth Factor in Wound Healing: Synergistic Effects with Other Growth Factors. Proceedings of the National Academy of Sciences of the United States of America, 84, 7696-7700. https://doi.org/10.1073/pnas.84.21.7696

[15] Mineo, A., Suzuki, R. and Kuroyanagi, Y. (2013) Development of an Artificial Dermis Composed of Hyaluronic Acid and Collagen. Journal of Biomaterials Science, Polymer Edition, 24, 726-740. https://doi.org/10.1080/09205063.2012.708190

[16] Prausnitz, M.R. (1999) A Practical Assessment of Transdermal Drug Delivery by Skin Electroporation. Advanced Drug Delivery Reviews, 35, 61-76. 
https://doi.org/10.1016/S0169-409X(98)00063-5

[17] Ueda, T., Sakai, K., Watanabe, Y. and Suzuki, H. (2009) Performance of Bipolar Electroporation Instrument and Its Improvement. Fragrance Journal, 37, 113-117. 